\title{
Neurological infection with SARS-CoV-2 - the story so far
}

\section{Tom Solomon (D)}

As the COVID-19 pandemic developed and neurological manifestations were reported, concern grew that SARS-CoV-2 might directly invade neuronal cells. However, research throughout the year to address this concern has revealed a different story with inflammatory processes at its centre.

Historically, the great epidemic causes of neurological disease, such as Japanese encephalitis and polio viruses, have directly infected the CNS. As the COVID-19 pandemic developed and increasing numbers of patients with neurological disease were reported, the question was, would SARS-CoV-2 behave in a similar way?

Initial reports of patients with COVID-19 who exhibited clinical evidence of brain inflammation suggested that SARS-CoV-2 encephalitis can occur ${ }^{1}$, though the rarity with which the virus was found in the cerebrospinal fluid (CSF) implied that immune-mediated damage is more important than viral replication in neurons. As the numbers of case reports and small series grew, it became clear that anosmia, encephalopathy and stroke were the predominant neurological syndromes associated with COVID-19 (REF.'.).

Anosmia and associated dysgeusia are common with SARS-CoV-2 infection and often occur in the absence of other symptoms. Herpes simplex virus 1 infects the olfactory bulb and subsequently the brain, leading to encephalitis; animal models have shown that the same is true for some coronaviruses, including SARS-CoV, which causes severe acute respiratory syndrome. Consequently, concerns were raised that olfactory infection with SARS-CoV-2 might lead to CNS disease. However, an elegant study published in July 2020 indicated that SARS-CoV-2 infects the supporting cells in the olfactory epithelium rather than the sensory neurons ${ }^{2}$.

In this study, single-cell RNA sequencing gene expression analysis of human nasal biopsy samples showed that the supporting cells, particularly sustentacular and horizontal basal cells, express angiotensin-converting enzyme 2 (ACE2) receptors and cell surface transmembrane serine protease 2 (TMPRSS2), both of which are critical for viral entry, whereas olfactory neurons do not. Immunohistochemical staining confirmed ACE2 protein expression in these same cells. Equivalent observations were made in the mouse, where the deeper olfactory bulb tissue could also be examined. Here, ACE2 receptors were found in vascular cells, predominantly pericytes, and immune cells of the macrophage/monocyte lineage, but not in neurons ${ }^{2}$.

SARS-CoV-2 infection of these supporting cells could lead to anosmia via several mechanisms. First, the supporting cells in the olfactory epithelium regulate local water and ion balance, and damage to them can influence signalling from the olfactory neurons to the brain. Second, infection of these cells and pericytes in the olfactory bulb could perturb neuronal signalling through local inflammation with cytokine release. Third, vascular damage and hypoperfusion in the olfactory bulb could contribute to impaired function. Finally, any of these changes could indirectly trigger death of the olfactory neurons. Imaging studies in patients with COVID-19 and anosmia have revealed hyperintensity and swelling of the olfactory bulb, consistent with inflammation, which subsequently resolved, as do the symptoms in most patients.

Anosmia is the most common neurological symptom in mild COVID but alterations in higher mental function are more important among patients who are hospitalized. Terms such as encephalopathy and delirium have been used to describe these changes; different specialities have preferred different terms, which hinders data comparisons.

In one study, 118 of 140 consecutive patients admitted to intensive care units (ICUs) with COVID-19 developed delirium with a combination of acute disturbances in attention, awareness and cognition; 88 had corticospinal tract signs ${ }^{3}$. Delirium can occur in any patient in ICU but accumulating data suggest we are seeing more than expected and that these symptoms could be characteristic of SARS-CoV-2 infection, especially given that delirium, encephalopathy and other neuropsychiatric manifestations are also seen in patients with milder COVID-19 and not in ICU.

Analysis of CSF, autopsy samples and imaging data is beginning to elucidate mechanisms that could underlie cognitive disturbances. Typically, pleocytosis is not seen in CSF of individuals with encephalopathic COVID-19, but protein levels can be elevated with matched oligoclonal bands. Elevated plasma and CSF levels of cytokines, glial fibrillary acidic protein and neurofilament light chain in COVID-19 are thought to reflect a proinflammatory systemic and brain response

\footnotetext{
Key advances

- Anosmia, encephalopathy and stroke are the most common neurological syndromes associated with SARS-CoV-2 infection ${ }^{1}$, though many others have been reported.

- Analysis of human biopsy samples suggests that anosmia results predominantly from SARS-CoV-2 infection of non-neuronal cells in the olfactory epithelium and olfactory bulb ${ }^{2}$, leading to local inflammation and neuronal malfunction.

- A high proportion of patients admitted to intensive care units with COVID-19 develop delirium, and evidence suggests that this is caused by microvascular and inflammatory mechanisms ${ }^{3}$.

- Autopsy data show activation of astrocytes and microglia in COVID-19, particularly in the brainstem, where there is also infiltration of cytotoxic T cells ${ }^{7,9}$.

- SARS-CoV-2 can be detected in the brain with PCR and immunohistochemistry, but the evidence to date suggests it is mostly in vascular and immune cells rather than directly infecting neurons ${ }^{7,9}$.
} 


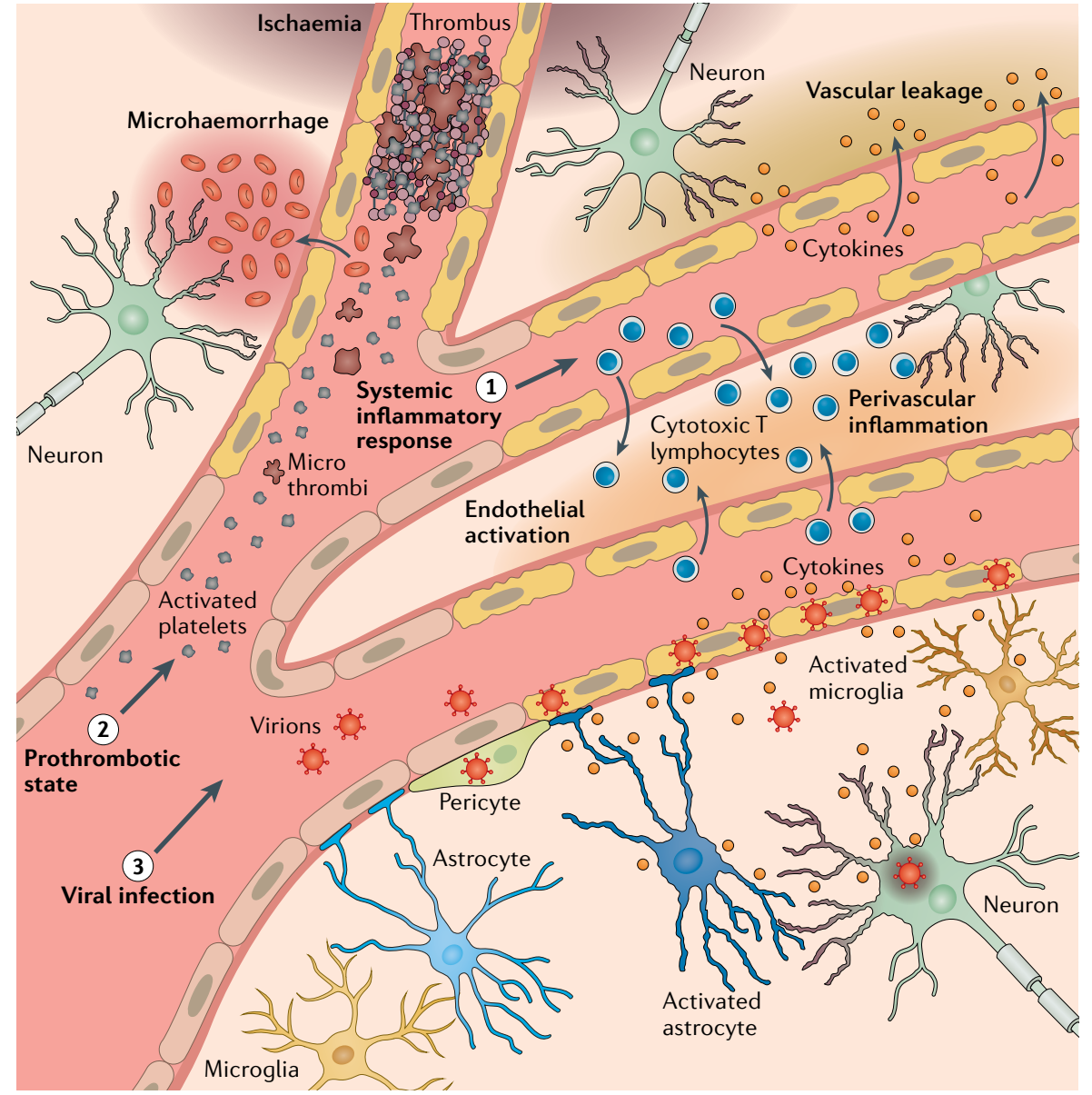

Fig. 1 | Current understanding of predominant COVID-19 neurological disease mecha-

nisms. Mechanisms include a systemic inflammatory response (1), a prothrombotic state (2) and direct viral invasion (3).

that involves microglial activation and subsequent neuronal damage $e^{4,5}$. Further evidence for inflammatory mechanisms comes from imaging findings, which showed meningeal enhancement and diffuse white matter abnormalities as well as microhaemorrhages ${ }^{3}$.

In addition to inflammatory changes, coagulopathy and vascular endothelial dysfunction, which cause large vessel strokes in COVID-19, can also lead to small vessel occlusions and microhaemorrhages, which could contribute to subtle neurological and neuropsychiatric presentations, as suggested by clinical and imaging studies ${ }^{6}$. However, the most definitive data on the underlying mechanisms come from autopsy series. One such study included 43 individuals, most of whom died in ICUs, general wards or nursing homes from pneumonia or sepsis associated with COVID-19 (REF.7). Six of these individuals had acute ischaemic brain lesions. Activation of astrocytes was widespread in the brain, whereas activation of microglia was confined to the brainstem and cerebellum. Cytotoxic $\mathrm{T}$ cells were also seen in the brainstem and in the meninges of many patients. RNA detection and immunohistochemistry suggested that SARS-CoV-2 was widely distributed throughout the brain, especially the brainstem. However, no correlation was seen between the location of the virus and inflammation, or indeed between PCR detection and immunohistochemical staining of the virus. Without double immunostaining, it is hard to be certain which cell types were infected, but results from an electron microscopy study indicate infection of vascular endothelial cells rather than neurons ${ }^{8}$. However, an autopsy study of 33 patients provided PCR and immunohistochemical evidence of SARS-CoV-2 in cells thought to be olfactory neurons, and in anatomically connected regions of the brain'. Nevertheless, some patients with encephalopathic changes respond to corticosteroids, underscoring the importance of immune-mediated mechanisms rather than direct viral effects ${ }^{10}$.

In summary, if the retina is the window on the brain, then for understanding SARSCoV-2, the nose has perhaps been the front door. For just as SARS-CoV-2 causes disturbance of smell without infecting olfactory neurons, evidence suggests that disturbance of higher mental function occurs predominantly without infection of CNS neurons (FIG. 1). Although the virus can enter the brain, it seems to predominantly infect vascular and immune cells. Local inflammation upregulates astrocytes and microglia, perhaps compounding the effects of circulating pro-inflammatory cytokines in severe systemic disease. Microvascular infarcts and haemorrhages, which are part of the systemic coagulopathy and vasculopathy of COVID-19, are probably also critical in the development of encephalopathy, delirium and other neurological manifestations of SARS-CoV-2 infection.

Tom Solomon (iD ${ }^{1,2}$

'Health Protection Research Unit in Emerging and Zoonotic Infections, National Institute for Health Research, Institute of Infection Veterinary and Ecological Sciences, University of Liverpool, Liverpool, UK.

${ }^{2}$ The Walton Centre NHS Foundation Trust, Liverpool, UK.

e-mail:tsolomon@liverpool.ac.uk https://doi.org/10.1038/s41582-020-00453-w

Ellul, M. A. et al. Neurological associations of COVID-19. Lancet Neurol. 19, 767-83 (2020). Brann, D. H. et al. Non-neuronal expression of SARS-CoV-2 entry genes in the olfactory system suggests mechanisms underlying COVID-19-associated anosmia. Sci. Adv. 6, eabc5801 (2020).

3. Helms, J. et al. Delirium and encephalopathy in severe COVID-19: a cohort analysis of ICU patients. Crit. Care 24, 491 (2020).

4. Kanberg, N. et al. Neurochemical evidence of astrocytic and neuronal injury commonly found in COVID-19. Neurology 95, e1754-e9 (2020).

5. Pilotto, A., Padovani, A. \& ENCOVID-BIO Network. Reply to the letter "COVID-19-associated encephalopathy and cytokine-mediated neuroinflammation". Ann. Neurol. 88, 861-862 (2020).

6. Beyrouti, R. et al. Characteristics of ischaemic stroke associated with COVID-19. J. Neurol. Neurosurg. Psychiatry 91, 889-891 (2020).

7. Matschke, J. et al. Neuropathology of patients with COVID-19 in Germany: a post-mortem case series. Lancet Neurol. 19, 919-929 (2020).

8. Paniz-Mondolfi, A. et al. Central nervous system involvement by severe acute respiratory syndrome coronavirus-2 (SARS-CoV-2). J. Med. Virol. 92, 699-702 (2020).

9. Meinhardt, J. et al. Olfactory transmucosal SARS-CoV-2 invasion as a port of central nervous system entry in individuals with COVID-19. Nat. Neurosci. https:// doi.org/10.1038/s41593-020-00758-5 (2020).

10. Pugin, D. et al. COVID-19-related encephalopathy responsive to high-dose glucocorticoids. Neurology 95, 543-546 (2020).

\section{Acknowledgements}

T.S. is supported by the European Union Horizon 2020 research and innovation programme ZikaPLAN (Preparedness Latin America Network; grant agreement No. 734584), the National Institute for Health Research (NIHR) Global Health Research Group on Brain Infections (No. 17/63/110) and the NIHR Health Protection Research Unit in Emerging and Zoonotic Infections at University of Liverpool in partnership with Public Health England (PHE), in collaboration with Liverpool School of Tropical Medicine and the University of Oxford (Grant No. NIHR200907). The views expressed are those of the author and not necessarily those of the NHS, the NIHR, the Department of Health or PHE. I am grateful to Avindra Nath for helpful discussions, and also for the support of colleagues in the UKRI COVID-19 Clinical Neuroscience Study (COVID-CNS), Liverpool Health Partners, and the Centre of Excellence in Infectious Disease Research (CEIDR), Liverpool.

\section{Competing interests}

The author declares no competing interests. 